\author{
GENERAL INFORMATION \\ https://www.cambridge.org/core/journals/ \\ canadian-journal-of-mathematics/information
}

The CMS publishes two research journals: the Canadian Journal of Mathematics (CJM) for longer papers, and the Canadian Mathematical Bulletin (CMB) for shorter papers. To be submitted to CJM, papers should be at least 18 pages long. Shorter papers should be submitted to the CMB. All submitted papers are considered subject to the understanding that they have not been published and that they are not being considered for publication elsewhere. To be accepted, papers must treat new mathematical research, be well written, and be of interest to a significant segment of themathematical community. Papers may be written in English or French. Manuscripts must be prepared in accordance with the instructions for authors provided at

https://www.cambridge.org/core/journals/canadian-journalof-mathematics/information/information-for-author

\section{SUBSCRIPTION INFORMATION}

Subscription rates can be found at

https://www.cambridge.org/core/journals/canadian-journalof-mathematics/subscribe

Members of the CMS are entitled to a reduced subscription rate. For more information, please contact the CMS at memberships@cms.math.ca.

\section{CANADA POSTMASTER}

Canadian Journal of Mathematics

Publications Mail Registration: 40005057

Send address changes to:

Journals Fulfillment Department, Cambridge University Press, One Liberty Plaza, New York, NY 10006 USA.

\section{CANADIAN JOURNAL OF MATHEMATICS}

(USPS \# 010-741) is published six times per year, bimonthly (February, April, June, August, October, December). Published by Cambridge University Press, One Liberty Plaza, New York, NY 10006 USA on behalf of the Canadian Mathematical Society. US Mailing Agent: Sheridan Press, 450 Fame Ave., Hanover, PA 17331 USA.

Periodicals postage paid at New York, NY and at additional mailing offices.

US POSTMASTER: Send address changes to Journals Fulfillment Department, Cambridge University Press, One Liberty Plaza, New York, NY 10006 USA.

\section{RENSEIGNEMENTS GÉNÉRAUX \\ https://www.cambridge.org/core/journals/ \\ canadian-journal-of-mathematics/information}

La SMC publie deux revues de recherche : le Journal canadien de mathématiques (JCM) qui présente des articles longs et le Bulletin canadien de mathématiques (BCM) des articles plus courts. Les articles destinés au JCM doivent compter au moins 18 pages. Les articles plus courts doivent être acheminés au BCM. Il est entendue que les articles présentés pour publication sont inédits et qu'ils ne sont pas à l'étude pour publication dans d'autres revues. Un article accepté porte sur des recherches récentes, est bien rédigé et s'avère susceptible d'intéresser bon nombre de mathématiciens. Nous acceptons les articles rédigés en langues anglaise ou française. Les articles doivent répondre aux conventions énoncés pour les auteurs au

https://www.cambridge.org/core/journals/canadian-journalof-mathematics/information/information-for-author

\section{ABONNEMENTS}

Les tarifs dabonnements sont disponibles au https://www.cambridge.org/core/journals/canadian-journalof-mathematics/subscribe

Les membre de la SMC ont droits à un taux d'abonnements réduits. Pour plus amples renseignements, veuillez contacter la SMC à adhesions@smc.math.ca.

\section{POSTES CANADIENNES}

Journal canadien de mathématiques

No. d'enregistrement du courrier publications: 40005057

Envoyer les changements d'adresses aux :

Journals Fulfillment Department, Cambridge University Press, One Liberty Plaza, New York, NY 10006 USA.

JOURNAL CANADIEN DE MATHEMATIQUES (USPS \# 010-741) est publié six fois par an, bimensuel (février, avril, juin, août, octobre, décembre). Publié parCambridgeUniversity Press, One Liberty Plaza, New York, NY 10006 USA au nom de la Société mathématique du Canada. Agent de poste US: Sheridan Press, 450 Fame Ave., Hanover, PA 17331 USA.

Postes payé à New York, et autres bureaux de diffusion supplémentaires.

POSTES AMÉRICAINES: Envoyer les changements d'adresses aux Journals Fulfillment Department, Cambridge University Press, One Liberty Plaza, New York, NY 10006 USA. 


\section{In this issue / Dans ce numéro}

$2021,73 / 3$

597

\section{2}

656

687

717

769
The Chord Index, its Definitions, Applications, and Generalizations

Boundedness of Differential Transforms for Heat

Semigroups Generated by Schrödinger Operators

Coxeter Diagrams and the Köthe's Problem

An Application of Spherical Geometry to Hyperkähler Slices

Extension Property and Universal Sets

Coisotropic Submanifolds in $b$-symplectic Geometry

On Restriction Estimates for the Zero Radius Sphere over Finite Fields

Reflection of Willmore Surfaces with Free Boundaries

Bounding Selmer Groups for the Rankin-Selberg

Convolution of Coleman Families

Classifying spaces for étale algebras with generators

Albert algebras over rings and related torsors
Zhiyun Cheng

Zhang Chao and

José L. Torrea

Ziba Fazelpour

and Alireza

Nasr-Isfahani

Peter Crooks and

Maarten van

Pruijssen

Łukasz Kosinski and Włodzimierz

Zwonek

Stephane Geudens, and Marco Zambon

Alex Iosevich, Doowon Koh, Sujin Lee, Thang Pham, and Chun-Yen Shen

Ernst Kuwert and Tobias Lamm

Andrew Graham, Daniel R. Gulotta, and Yujie Xu

Abhishek Kumar

Shukla and

BenWilliams

Seidon Alsaody 